\title{
Advantage of knowing nature's secrets
}

Sir - The epoch-making results of Wilmut et al. ${ }^{1}$ describing the cloning of adult sheep once more illustrates the perpetual problem of equating scientific endeavour with the public interest. I noted with interest the media frenzy which greeted these findings together with the subsequent public discussion of the likelihood and implications, both practical and moral, of future cloning of adult humans. We scientists are intrigued and driven by every new discovery no matter how small. We also, however, have an obligation to relay this information to the public in a manner that creates a suitable environment for informed discussion to take place.

In this respect, I was reminded of the cautionary words of Pierre Curie on the occasion of his address to the Swedish Academy of Sciences in 1903 when he and Marie Curie received the Nobel Prize for Physics: "...one can ask if humanity is at an advantage in knowing nature's secrets, if it is mature enough to make use of them or if this knowledge might not be harmful to it"'. I, for one, agree with Nature's decision to publish the results of Wilmut et al., and concur with Curie's conclusion that "...humanity will derive more good than bad from new discoveries"2.

Michael J. Taggart

Physiological Laboratory,

University of Liverpool,

Liverpool L69 3BX, UK

Sir - Your recent News article ${ }^{3}$ on the reaction to sheep cloning suggests that "scientists agree that cloning of humans would be unethical". Indeed, a certain Jeremy Rifkin goes so far as to suggest that penalties for human cloning should be on a par with those for "rape, child abuse and murder".

There are two issues that Rifkin and his band of crusaders should bear in mind. First, history shows that scientific knowledge is used and developed, no matter what ethical considerations are involved. For example, despite the many cries to "Ban the Bomb", nuclear weapons have been developed by all the major (and some minor) powers. Second, one could imagine a situation in which human cloning might be the only option available to recover a human life. Should Rifkin deny the option of cloning to the parents of a young child fatally injured in an accident, if viable cells could be recovered from the body?

I am not aware of any conclusive poll that confirms that "scientists agree that cloning of humans would be unethical". Moreover these issues are too important to be left to the champions of the knee-jerk reaction highlighted in your news article. Mike Fainzilber

Molecular Neurobiology Laboratory,

Karolinska Institute BZ-Lab.,

Doktorsringen 12A,

S17177 Stockholm, Sweden

e-mail:michael@cajal.mbb.ki.se

Sir - The publication of the experimental protocol through which Dolly came to life ${ }^{1}$ created a considerable stir in the media. In this context, the arguments put forward in your leading article $\mathrm{i}^{4}$ favour of a mere moratorium rather than total and definitive banning of this type of experiment in humans are not acceptable. More precisely, it is unacceptable to let people think that in certain circumstances human beings could be brought to life by means of somatic cell cloning, because the essence of humanity lies in the uniqueness of each of its members, resulting from the unique recombination of two equally unique genomes.

In consequence, the only solution is to pronounce a universal ban on human cloning. When this is done - and only then - can one envisage a one-year moratorium to define the conditions in which cloning experiments involving human cells could be performed, but for the sole advance of knowledge and the ultimate prospect of growing in vitro differentiated cells or organs intended for transplant therapy. Francis Galibert

University of Rennes 1 ,

UPR 41,

2 avenue du professeur Leon Bernard,

35043 Rennes Cedex, France

Jacqueline Godet

Centre de Génétique

Moléculaire et Cellulaire,

CNRS/University of Lyon,

69622 Villeurbanne, France

Jean-Claude Kader

Laboratoire de Physiologie

Cellulaire et Moléculaire,

URA CNRS 2135, Université Paris 6,

75252 Paris Cedex 05, France

Jean-Antoine Lepesant

Institut Jacques Monod,

CNRS/Université Paris 7,

75251 Paris Cedex 05, France

Sir - In the Human Fertilisation and Embryology Act 1990 and the Animal (Scientific Procedures) Act 1986, the United Kingdom has a regulatory framework which was already in place and which was applied to the work on the cloning of sheep at the Roslin Institute.

Nature has reported accurately and systematically the work of the House of Commons Select Committee on Science and Technology, and in particular our report on human genetics, in which we recommended the setting up of the Human Genetics Advisory Commission as a standing body to report publicly on all aspects of human genetics and its applications, including ethical, legal and social aspects. The commission was meeting for the first time on the very day Nature published the Letter reporting the results of the successful cloning of a sheep ${ }^{1}$.

It was therefore disappointing that your leading article 4 concluded by saying it "is shaming" for "politicians only now to be requesting guidance about what appears in today's Nature".

We are however confident that our further current inquiry into cloning, and the work of our successor committee in the next parliament, will continue to be well reported in Nature. It is an important channel of communication not only within science, but also with governments and politicians about science and its impact on society.

Giles Shaw

(Chairman)

Jeremy Bray

Select Committee on Science and Technology, House of Commons,

London SWIA OAA, UK

- The leading article actually refers to "a US president and other politicians...”. - Editor, Nature.

Sir - I write to comment on the picture of Dolly, the cloned sheep, on the cover of the 27 February issue. Superficial inspection of this photograph reveals that Dolly unexpectedly has one black hoof. Could it be that the cytoplasm of the enucleated Scottish Blackface egg contains unknown genetic information that specifies the left rear hoof? Or, is there some other Photoshop-like explanation for this maternally derived appendage (see Figure 2, page 812$)$ ?

The possibilities are especially interesting coming on the heels (hooves?) of the recent report of a fowl-up when quail neural cells are transferred into chicken brains.

Sidney Strickland

University at Stony Brook,

Stony Brook, New York 11794-8651, USA.

e-mail: strickland@pharm.sunysb.edu

1. Wilmut, I. et al. Nature 385, 810-813 (1997).

2. Quinn, S. Marie Curie. A story (Mandarin, UK, 1995).

3. Masood, E. Nature 385, 757 (1997).

4. Nature 385, 753 (1997). 